\title{
A TEM Based weak Signal Extraction Method of MRS Under Strong Noise in Tunnel
}

\author{
Wei Zhao, Wentao Liu, Xiu Li \\ College of Geology Engineering and Geomatics, Chang'an University \\ Xi'an, China
}

\begin{abstract}
Underground magnetic resonance sounding (MRS) low signal-noise ratio (SNR) weak signal is hard to manage with traditional de-noising method such as fitting and filter. In view of this, this article makes use of both transient electromagnetic method (TEM) data and MRS data obtained from integrated detecting equipment. The goal is to extract weak MRS signal based on TEM under strong background noise. At first, underground electrical distribution can be acquired from TEM data, which is processed by equivalent conductive plane method. Then, the initial model of moisture content distribution was constructed on geological information and 3D MRS forwarding was made with finite element method. At last, the correlation coefficient series and deviations between forwarding data and measured data were calculated. The initial model would be corrected until the result is satisfying. Model calculation shows that this method can effectively extract the weak nuclear magnetic signals in tunnel under strong background noise.
\end{abstract}

Keywords-MRS; weak signal; TEM

\section{INTRODUCTION}

With the rapid development of "the Belt and Road" construction, a large number of long tunnels are going to be built. However, construction progress always be delayed and workers lives always be threatened because of potential pernicious water. It's urgent to find a new way to forecast the potential dangers in tunnel. MRS method had achieved good results in water resource exploration as a searching groundwater directly method (Jun et al. $2011^{[1]}$ ). To solve the problem of water detection in tunnels, Lin Jun proposed MRS method to underground engineering field for the first time (Jun et al. 2013). However, study shows that underground MRS signal is weaker with lower signal to noise ratio, shown in Fig. 1. Traditional de-noising method such as fitting and filtering are helpless. This method makes use of both TEM data and MRS data obtained from integrated detecting equipment. The goal is to extract weak MRS signal under strong background noise. Overall, this paper provides a new method to deal with weak MRS signals in tunnel.

\section{THEORY AND METHOD}

The basic idea of this method was shown in Fig. 2. First of all, underground resistivity distribution can be obtained through equivalent conductive plane method based on TEM data. The initial model of aquifer was acquired considering different lithology relationships between resistivity and moisture content, which is estimated combine with geological information. Then, 3D MRS forwarding was calculated on the initial model. The distribution of transmitting magnetic field was calculated by finite element method directly. Finally, the calculation of correlation and deviation between forward data and measured data was used as evaluation criterion. The initial model will be modified all the time until correlation and deviation results are satisfying.

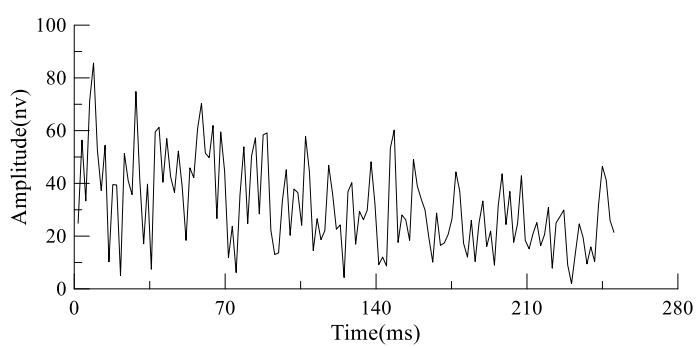

Fig.1. MRS measured signal in tunnel

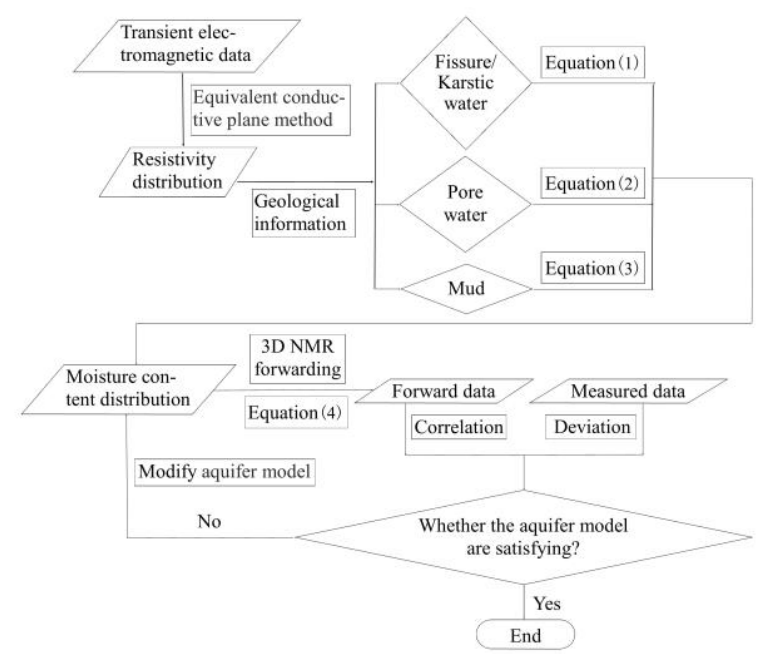

Fig.2. Signal extraction process

Deduction from resistivity distribution to moisture content distribution is a critical procedure of this method. In order to obtain a good initial model, geological information must be sufficiently considered.

For the situation that the abnormal bodies in front of the tunnel are underground watercourse or pore water (Xuan, 2012). Groundwater resistivity is closely connected with geological parameters such as mineralization, porosity et al. 


$$
\log \rho_{w}=a+b \log C_{k}
$$

Where $a$ denotes a coefficient connected with temperature and $b$ is a constant of $-0.86 ; \rho_{w}$ denotes ground water resistivity and $C_{k}$ denotes ground water mineralization.

$$
\rho_{s}=a \cdot \varphi^{-m} \cdot \rho_{w}, \quad \varphi=\frac{n_{w}}{100 P+n_{w}-n_{w} P}
$$

Where $a$ and $m$ are coefficients connected with lithology and cementation degree separately; $\rho_{s}$ and $\rho_{w}$ denote rock and ground water resistivity separately; $\varphi$ denotes porosity; $n_{w}$ denotes moisture content and $P$ is the density ratio of water and rock.

For the situation that the abnormal bodies in front of the tunnel are mud ( $\mathrm{Li}$ et al, 2012). Groundwater resistivity is closely connected with geological parameters such as formation factor, saturation et al.

$$
\frac{1}{\rho_{t}}=\frac{S_{w}^{n}}{F}\left(\frac{1}{\rho_{w}}+B \times Q_{v} / S_{w}\right), \quad S_{w}=\frac{S_{w \max }}{1+a \times e^{b \times n_{w}}}
$$

Where $\rho_{t}$ and $\rho_{w}$ denote mud and formation water resistivity separately; $F$ and $n$ denote formation factor and saturation exponent separately; $S_{w}$ denotes water saturation; $B$ denotes the equivalent conductivity of exchange cation and $Q_{v}$ denotes cation exchange capacity of unit volume; $S_{w \max }$ denotes maximum water saturation; $n_{w}$ denotes moisture content; $a$ and $b$ are coefficients.

Once we know some geological information in front of tunnel. The initial moisture content distribution model could be obtained with the help of resistivity distribution and geological information. 3D MRS forward data could be calculated.

$$
\begin{aligned}
& E_{0}(q)=\int_{v} K_{3 D}(q, \rho(\boldsymbol{r}), \boldsymbol{r}) \cdot n(\boldsymbol{r}) d v \\
& K_{3 D}(q, \rho(\boldsymbol{r}), \boldsymbol{r})= \frac{2 \pi f_{L} M_{0}}{I_{0}} B_{\perp}(\rho(\boldsymbol{r}), \boldsymbol{r}) \\
& \cdot \sin \left(\frac{1}{2} \gamma \frac{B_{\perp}(\rho(\boldsymbol{r}), \boldsymbol{r})}{I_{0}} q\right)
\end{aligned}
$$

In which, $K_{3 D}(q, \rho(\boldsymbol{r}), \boldsymbol{r})$ denotes 3D kernel function; $n(\boldsymbol{r})$ denotes moisture content at point $\boldsymbol{r}(x, y, z) ; f_{L}$ denotes larmor frequency; $M_{0}$ denotes hydrogen nuclear magnetization; $I_{0}$ denotes current; $B_{\perp}$ denotes excite field; $\rho(\boldsymbol{r})$ denotes resistivity magnitude at point $\boldsymbol{r}(x, y, z) ; \gamma=2.675 \times 10^{8} s^{-1} T^{-1}$ denotes hydrogen proton magnetic rotation ratio; $q=I_{0} \cdot \tau$ denotes pulse moment and $\tau$ denotes pulse duration.

\section{Model CAlculation}

In order to test weak signal extraction effect of this method. We made a tunnel simulation model. A cube of porous water filling model is designed in front of the tunnel face, as shown in Fig. 3. The cube magnitude is $10 \times 10 \times 3 m$, resistivity is $10 \Omega \cdot m$, moisture content is $50 \%$ and buried depth of $10.5 m$. The resistivity of surrounding rock is set to $100 \Omega \cdot m$. The transient electromagnetic data was simulated with 3D time domain finite difference forward modeling. The apparent resistivity was converted with equivalent conductive plane method, as show in Fig. 4.

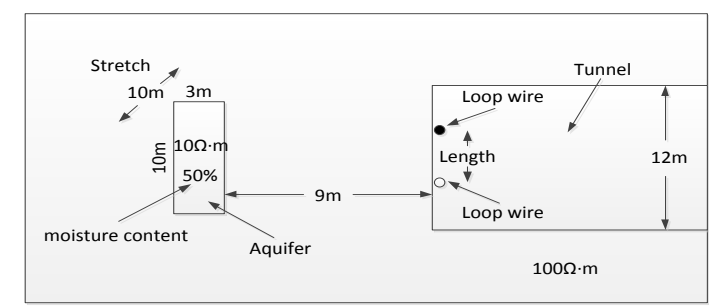

Fig.3. Water filling model

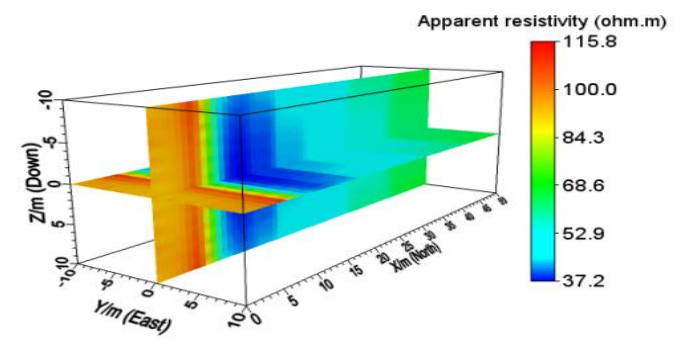

Fig.4. Apparent resistivity slice map

For the porous water filling model, we make use of (2) to get the initial moisture content distribution. Fig. 5 and Fig. 6 show the water content slice map. The corresponding parameters $a, \mathrm{~m} 、 \rho_{w}, P$ in (2) were set to $0.2,1.8,30$ and 0.4 .

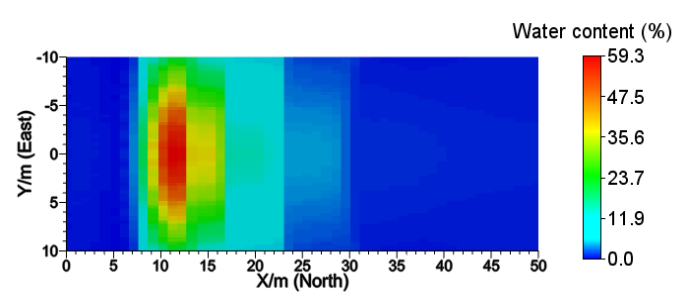

Fig.5. Moisture content slice map (xoy plane)

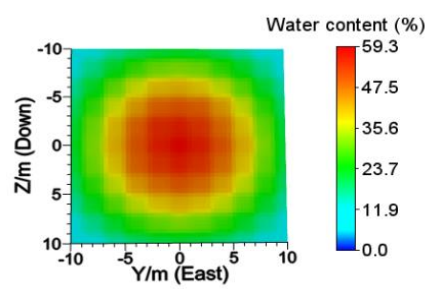

Fig.6. Moisture content slice map (yoz plane) 
For the purpose of testing the extraction result more directly, at first, 3D NMR forward modeling of different pulse moment under real model were made (the red lines shown in Fig. 7). A strong gaussian noise, $\mathrm{SNR}=2$, was added to the forward signal to get simulated measured data. Then, we made 3D NMR forward modeling under initial model and calculated the correlation and deviation between measured data and forward data. The initial water content model would be modified until the correlation and deviation result are satisfying. The signal final extraction results were shown as blue lines in Fig. 7.
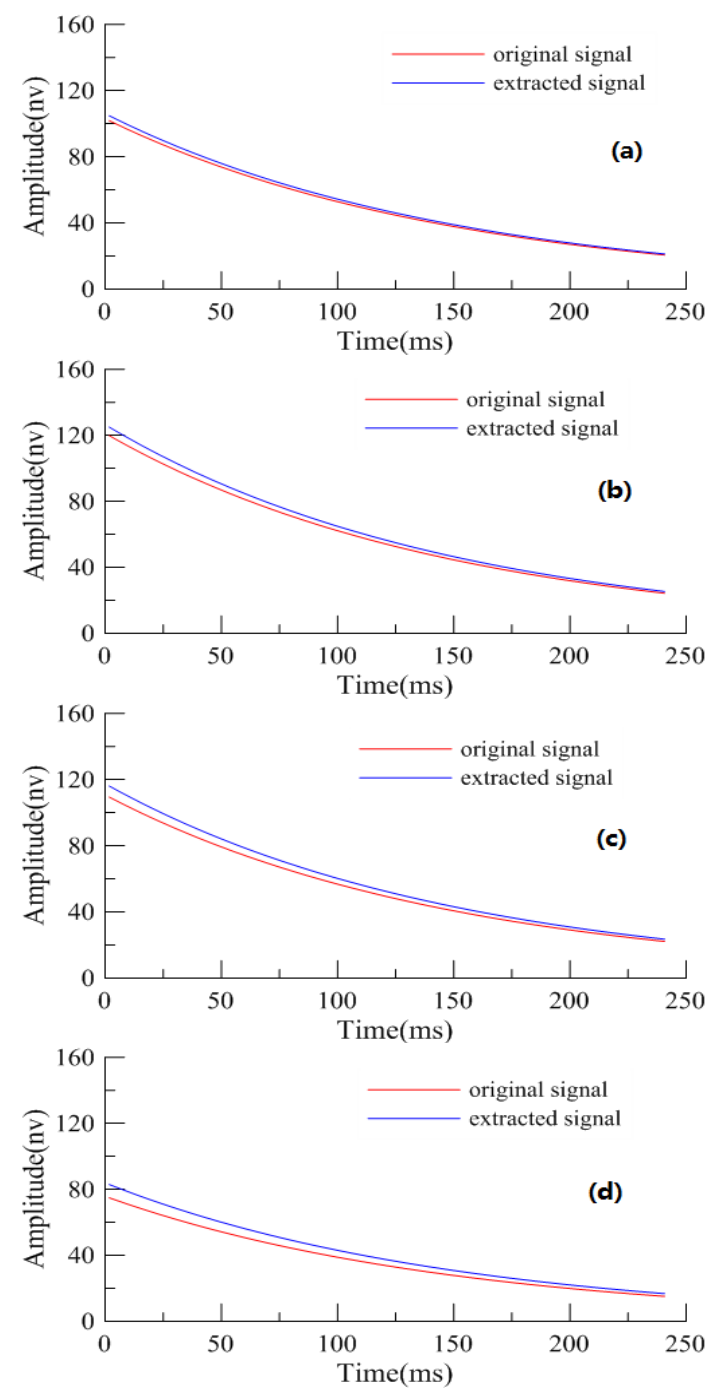

Fig.7. The original signals and extract results under different pulse moments (a)100A.ms; (b)150A $\mathrm{ms}$; (c)200A $\mathrm{ms}$; (d)250A $\cdot \mathrm{ms}$

\section{CONCLUSION}

(1) This paper puts forward a new method which could extract weak NMR signals under strong noise in tunnel effectively. The moisture content information could be get roughly at the same time.

(2) Deduction from resistivity distribution to moisture content distribution is a critical procedure of this method. The geological information must be sufficiently considered.

\section{REFERENCES}

[1] J. Lin, Q. M. Duan, Y. J. Wang, 2011, Theory and Design of magnetic resonance sounding instrument for ground-water detection and its application: Science Press. 